\title{
Calculation of secondary neutron fields generated by high-energy heavy-ion reactions using the Monte-Carlo code PHITS
}

\author{
H. Iwase*, D. Schardt, K. Gunzert-Marx+, E. Haettner \\ GSI, Planckstr. 164291 Darmstadt, Germany \\ ${ }^{+}$present address: Siemens Medical Solutions, 91052 Erlangen, Germany E-mail: \\ H.Iwaselgsi.de
}

\section{K. Niita}

RIST, Tokai 319-1106, Japan

E-mail: niita@tokai.rist.or.jp

\begin{abstract}
The neutron fields generated by high-energy heavy ions in a production target are of key importance as they are used as source terms for further transport calculations. Various calculations of secondary neutron fields generated by high-energy heavy-ion reactions, such as benchmarks with systematic data, neutron energy spectra and doses compared with data measured recently in GSI, and calculations for the Super-FRS project, are done. Comparison of measured neutron fields with calculations performed by the PHITS code will be included for more precise predictions for secondary neutron productions.
\end{abstract}

International Workshop on Fast Neutron Detectors

University of Cape Town, South Africa

April 3 - 6, 2006

\footnotetext{
* Speaker.
} 


\section{Introduction}

Secondary neutrons are most abundantly produced in high-energy heavy-ion reactions. However it is difficult to calculate the production rate and energy spectra due to complex reaction mechanisms. The Particle and Heavy Ion Transport code System PHITS is therefore employed for such calculations and further various applications.

\section{Benchmark calculation}

A benchmark calculation of the PHITS code was done by comparing with systematic data of secondary neutrons emitted in nucleus-nucleus reactions at energies from $100 \mathrm{MeV} /$ nucleon to 800 $\mathrm{MeV} /$ nucleon[2]. The data used in the benchmark were measured by two groups, Kurosawa et al.[3] and Iwata, Heilbronn, et al.[ $₫$, at HIMAC, Japan. The comparison shows that agreements between measured data and PHITS calculations are in general rather well for most cases.

Other comparison works using PHITS on secondary neutrons from heavy-ion reactions were carried out for thicker shielding systems[5]. Sasaki et al. measured neutron energy spectra at HIMAC, NIRS Japan with changing thickness of the shielding which placed behind a $5 \mathrm{~cm}$ copper target bombarded by $400 \mathrm{MeV} / \mathrm{u}$ carbon beams[6]. At Cave-A GSI Germany Wiegel and Fehrenbacher et al. performed shielding experiment. The NEMUS[7] (Neutron Multisphere Spectrometer) by PTB Germany was employed to detect energy spectra of neutrons at several measuring point in and outside the cave[8]. The considerable good agreement between both data and PHITS calculations were obtained[5].

\section{Recent neutron measurements at GSI and comparison}

\subsection{Neutron energy spectra from ${ }^{3} \mathrm{He}$ on water}

Energy spectra of secondary neutrons from $200 \mathrm{MeV} / \mathrm{u}{ }^{3} \mathrm{He}$ beams on $22.3 \mathrm{~cm}$ water target was measured in Cave-A GSI. The measurement was a part of a ${ }^{3} \mathrm{He}$ experiment for the purpose to obtain basic data set for a ${ }^{3} \mathrm{He}$ ion cancer therapy. Neutrons were detected using a $\mathrm{BaF}_{2}$ crystal scintillator. Events of secondary charged particles were discriminated from the data by seeing data scored by a plastic scintillator which placed in front of the $\mathrm{BaF}_{2}$ detector. Then measured time of flight data were converted to energy spectra. The efficiency of the $\mathrm{BaF}_{2}$ from Gunzert-marx et al.[9] was used. Fig.1] is a preliminary result of the experiment together with a PHITS calculation. It shows a discrepancy at 0 degrees, but for other angles data and calculations agree quite good as shown also in the figure for 30 degrees. The tendency was also shown in previous comparisons[2].

\subsection{Neutron dose from ${ }^{12} \mathrm{C}$ on water}

One of the recent study on ${ }^{12} \mathrm{C}$ cancer therapy is to evaluate accurate contributions of secondary fragments to the treatment dose. Therefore a systematic measurement for neutron dose has been carried out in GSI. Spatial neutron dose distributions around a water target bombarded by ${ }^{12} \mathrm{C}$ with different energies of $100 \sim 246 \mathrm{MeV} / \mathrm{u}$ were measured in Cave-M GSI using the WENDI[10] dosimeter, which includes tungsten moderator inside and has an improved energy response up to 5 GeV. 


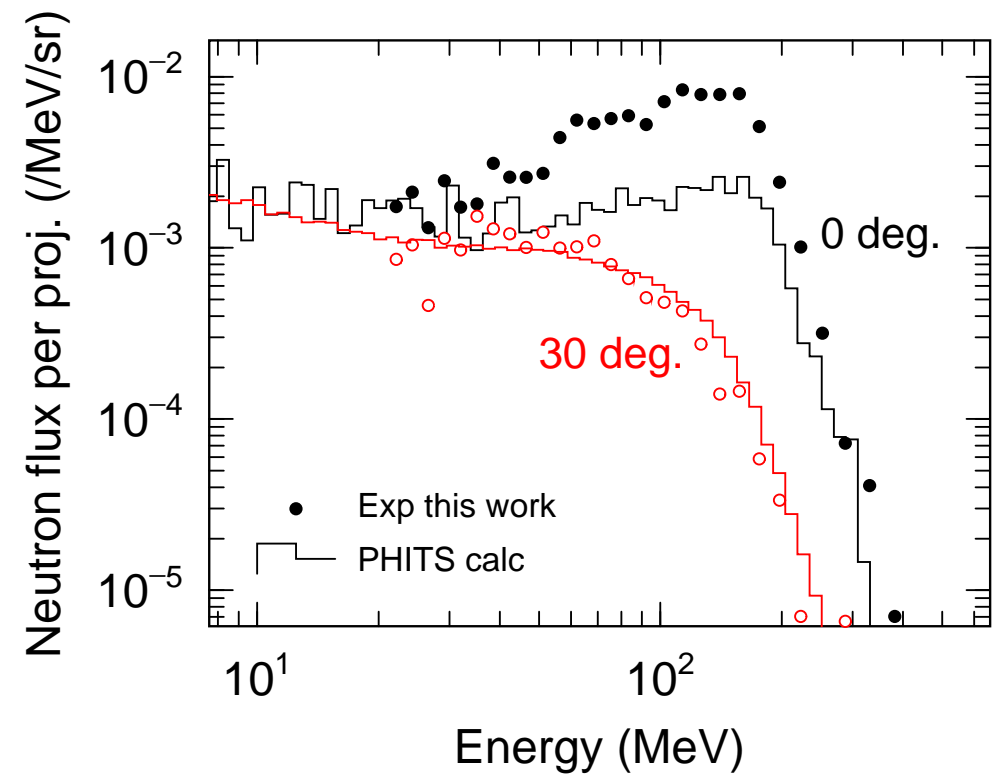

Figure 1: Comparison of measured (preliminary) and calculated neutron energy spectra from $200 \mathrm{MeV} / \mathrm{u}$ ${ }^{3} \mathrm{He}$ on $22.3 \mathrm{~cm}$ water target

Figure 2 shows measured neutron dose distributions (upper) and a comparison with other data (lower). On the upper figure one can see strong forward peaks of neutron doses and the tendency is prominent for higher projectile energies. On the lower of Fig. 2 the replotted data of $200 \mathrm{MeV} / \mathrm{u}$ is compared with data, which was originally neutron energy spectra measured by Gunzert-Marx et al.[12] using the $\mathrm{BaF}_{2}$ detector with the same beam and target and was afterward converted into dose with the neutron dose conversion factor[11]. It is noted that in the present data of WENDI contains signals which were caused by charged particles produced in the target. The contribution was experimentally confirmed in this study but could NOT be excluded from data. The discrepancy between both data at 0 degrees probably comes from the contribution by charged particles in the WENDI data, but otherwise both measured neutron doses agree well even these were measured with completely different methods. There is rather a large discrepancy between data and the calculation but all results agree within factor of three.

\section{Recent application of PHITS}

\subsection{Calculation of radiation field for the rare isotope production facility Super-FRS}

The Super-FRS[13], a next-generation high-intensity fragment separator, is being developed at the international project FAIR at GSI Germany. Rare isotopes of all elements up to uranium can be produced and spatially separated by the magnet system. In Super-FRS $1.5 \mathrm{GeV} /$ nucleon ${ }^{238} \mathrm{U}$ will be used as projectile with an intensity of $10^{12}$, therefore a lot of fragmentation is occurred at the production target and beam-dumps settled between dipole magnets. Especially secondary neutrons, most of which are produced with same velocity of projectile, i.e. with high-energy, can penetrate remarkably deep to the system and cause problems of heat-loads to the separator magnets, 

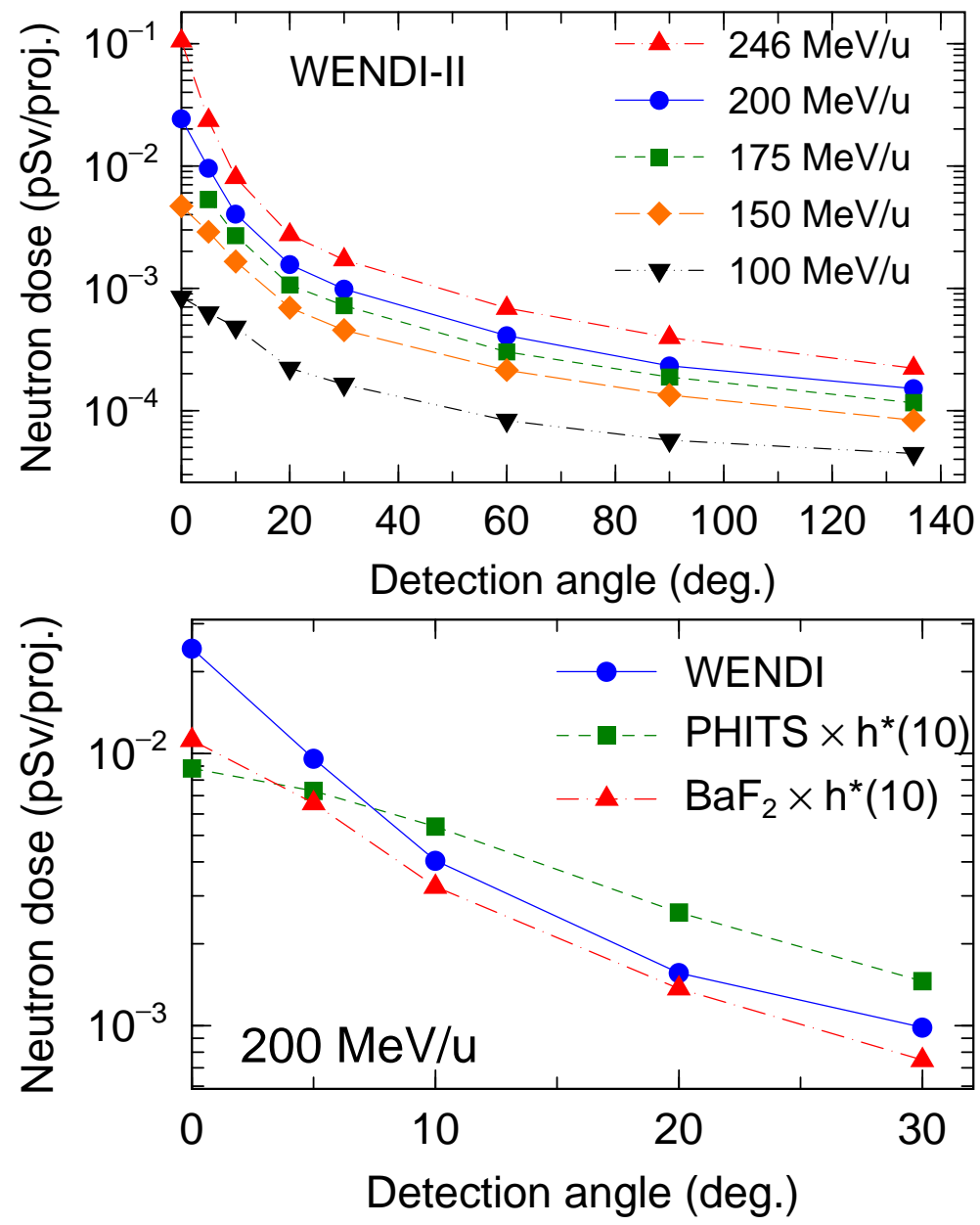

Figure 2: Measured neutron dose at $3 \mathrm{~m}$ distance from a $12.8 \mathrm{~cm}$ thick water target for different ${ }^{12} \mathrm{C}$ energies (upper) and comparison with data converted by neutron spectra by Gunzert-Marx[12] and calculations for $200 \mathrm{MeV} / \mathrm{u}$ (lower)

activation of the material, high dose beyond shielding, and so on. Therefore the PHITS code has been used in the project for the design.

The calculated results had been reported in different papers. Around the target position, neutron fields, heat-loads on system, and induced dose beyond shielding were calculated with modeling the geometry as accurate as possible[13].

Because of no data and routines to calculate activations in the PHITS code, a model was newly developed[14]. Activations of produced all fragments are calculated and for each gamma-ray with different energy an induced dose is calculated at any point. In addition, attenuation of gamma-ray in matter including self-shield of activated material is calculated. In the way 3-dimensional dose distributions induced by activations can be calculated very precisely using the model. Around the beam-dump position of the Super-FRS, neutron fields, activations, and induced dose were calculated[13]. 


\section{Summary}

Due to complex reaction mechanisms there were a certain limitation by simple models to calculate production and transport of secondary neutrons by heavy ions. The PHITS code was therefore developed with combining various physical and semi-empirical models and data. Benchmark calculations were performed on secondary neutron productions from various combinations of projectile with different energies and target with different thickness, and reasonably good agreements were obtained. The PHITS code is recently applied for various experiments and designs. In this study comparison between neutron measurement in GSI and PHITS calculations, and PHITS calculations for the international project FAIR were presented. The development of the PHITS code has been continued and improvements and additional models will be included for more precise predictions for secondary neutron productions.

\section{References}

[1] H. Iwase, K. Niita and T. Nakamura, Development of General-purpose Particle and Heavy ion Transport Monte Carlo code, Journal of Nucl. Sci. and Technol., Vol. 39, No. 11, p 1142-1151 (2002) ; K. Niita, et al. Particle and Heavy Ion Transport Code System; PHITS Proc. 2003 Symposium on Nuclear Data, Nov. 2003, JAERI, Tokai

[2] H. Iwase, Development and Experimental Evaluation of a General-Purpose Heavy-Ion Transport Monte Carlo Code, Ph. D. thesis, Tohoku University, Japan, March 2003.

[3] T. Kurosawa, N. Nakao, T. Nakamura, U. Uwamino, T. Shibata, N. Nakanichi, A.Fukumura, and K. Murakami, Nucl. Sci. and Eng., 132, 30 (1999); T. Kurosawa, N. Nakao, T. Nakamura, Y. Uwamino, T. Shibata, A. Fukumura, and K. Murakami, Journal of Nucl. Sci. and Technol., 36-1, 42 (1999); T. Kurosawa, T. Nakamura, N. Nakao, T. Shibata, Y. Uwamino, and A. Fukumura, Nucl. Instr. and Meth. , A430, 400 (1999); T. Kurosawa, N. Nakao, T. Nakamura, H. Iwase, H. Sato, Y. Uwanimo, and A. Fukumura, Phys. Rev. , C62, 044615 (2000).

[4] Y. Iwata, L. Heilbronn, H. Sato, K. Niita, et al., Phys. Rev. , C64, 054609 (2001).

[5] H. Iwase B. Wiegel, G. Fehrenbacher, et al., Radiat. Prot. Dosimetry, 116, No. 1-4, 640-646 (2005).

[6] M. Sasaki, N. Nakao, T. Nunomiya, et al., Nucl. Instrum. Methods Phys. Res. B, 196, 113-124 (2002).

[7] B. Wiegel and A. V. Alevra, Nucl. Instrum. Methods Phys. Res. A, 476, 36-41 (2002).

[8] G. Fehrenbacher, B. Wiegel, H. Iwase, et al., GSI Preprint 2004-06 (2004)

[9] K. Gunzert-Marx, D. Schardt, R.S. Simon, et al., Nucl. Instrum. Methods Phys. Res. A, 536, 146-153 (2005).

[10] R. H. Olsher, H. H. Hsu, A. Beverding, et al., Health Phys., 79, 170-81 (2000).

[11] ICRP 64: ICRP Publ. 74, Pergamon Press, (1996).

[12] K. Gunzert-Marx, Ph-D Thesis, TU Darmstadt Germany (2003).

[13] H. Geissel, M. Winkler et al., Technical report on the design, construction, comissioning and operation of the Super-FRS of FAIR, GSI (2005).

[14] H. Iwase, H. Weick, T. Radon, et al, Proceeding of AccApp05, in press 